\title{
Linearized method for the study of transverse instabilities driven by electron clouds
}

\author{
Giovanni Iadarola $\odot$, Lotta Mether®, Nicolas Mounet $\odot$, and Luca Sabato® \\ CERN, 1211 Geneva, Switzerland
}

(Received 2 July 2020; accepted 10 August 2020; published 26 August 2020)

\begin{abstract}
We present a linearized method to study transverse instabilities due to electron clouds. It is based on an accurate and compact characterization of the cloud dipolar and quadrupolar forces, that can be easily obtained from quick single-pass numerical simulations. The long-term stability properties of the bunch are then predicted by solving the linearized Vlasov equation, taking into account the dipolar forces introduced by the e-cloud along the bunch as well as the betatron tune modulation with the longitudinal coordinate due to the e-cloud quadrupolar forces. The method is benchmarked against macroparticle simulations based on the same characterization of the e-cloud and the results are compared against conventional "brute-force" simulations based on the Particle-In-Cell method. The effect of transverse nonlinearities due to the e-cloud, which are neglected by the linearized method, is also analyzed.
\end{abstract}

DOI: 10.1103/PhysRevAccelBeams.23.081002

\section{INTRODUCTION}

In particle accelerators, secondary electron emission and photoemission at the surface of the vacuum chambers can drive an exponential multiplication of low-energy electrons, leading to the formation of an electron cloud (e-cloud). E-clouds have been observed in several synchrotrons operating with positively charged particles, resulting in detrimental effects on the beam quality and on the performance of different accelerator systems [1-4].

Notably, e-clouds can drive transverse head-tail instabilities of the bunches, which cannot be suppressed by conventional transverse feedback systems due to the strong intrabunch motion. These instabilities have hampered the operation of different machines, including, recently, the Large Hadron Collider (LHC) [5-7].

Predictions of the beam stability in the presence of an e-cloud typically rely on macroparticle codes that simulate turn after turn the coupled dynamics of the beam and of the cloud distributions using the particle-in-cell (PIC) method. This approach has the advantage of allowing a detailed modeling of complex features of the e-cloud dynamics, but has the drawback of being extremely demanding in terms of computing resources and calculation time, which can become prohibitive when simulating instability timescales beyond a few seconds [8].

\footnotetext{
"giovanni.iadarola@cern.ch
}

Published by the American Physical Society under the terms of the Creative Commons Attribution 4.0 International license. Further distribution of this work must maintain attribution to the author(s) and the published article's title, journal citation, and DOI.
On the other hand, in the study of more conventional instabilities driven by electromagnetic coupling of the beam with surrounding structures, it is standard practice to characterize the short-term response of the environment to the beam distribution through effective quantities, namely wakefields and impedances. This proves to be extremely convenient as it allows predicting the long term behavior of the beam using fast macroparticle simulations or analytical approaches [9].

Analytical predictions for instabilities caused by impedance effects are often based on the linearized Vlasov equation $[9,10]$, and consist in identifying all the characteristic eigenmodes of the beam motion and calculating, for each of them, the corresponding growth rate and betatron frequency shift. This is done without the need for simulating directly the dynamics of individual particles. The advantage of this approach with respect to "brute-force" macroparticle simulations is twofold: first, as the computation time is not directly related to the growth rate of the instability, this method allows the study of slow instabilities that are out of reach for macroparticle simulations; second, the analysis of the eigenmode frequency shifts as a function of parameters of interest (e.g., bunch intensity, synchrotron frequency, chromaticity) provides important insight on the underlying mechanisms driving the instability, which in turn gives valuable information toward the identification of possible mitigation strategies.

Applying existing Vlasov methods to e-cloud instabilities is not straightforward due to certain characteristic features of the e-cloud, notably [5]: (i) the dipolar forces introduced by the e-cloud in response to a transverse distortion along the bunch cannot be modeled by conventional effective quantities (wakefields, impedances) for 
which Vlasov methods are routinely applied, but require a more general description, as clearly shown in [11]; (ii) the e-cloud introduces a modulation of the betatron tune as a function of the particle longitudinal coordinate along the bunch, which is known to have an impact on the beam stability properties [12].

A first attempt at using the Vlasov approach on e-cloud instabilities was made by Ohmi et al. in Ref. [13]. It was based on the approximation of the e-cloud response with a conventional broad-band resonator wakefield and considered the simplified case of a single radial mode. The tune modulation along the bunch introduced by the e-cloud was neglected. Perevendentsev extended these results in Ref. [14] by considering multiple radial modes and by introducing the effect of a linear dependence of the tune on the longitudinal particle position. Still such a model does not allow to fully describe the realistic behavior of the cloud. In particular it does not account for the dependence of the betatron tune on the longitudinal action, which, as we will show in the following, plays a significant role in defining the characteristics of the instability. Perevendentsev in Ref. [14] also proposes to describe the dipolar forces from the e-cloud with a generalized impedance (function of two variables) and derives expressions for the mode coupling matrix based on this description. To our knowledge this interesting line of research was not continued and no results from the application of this method are available in the literature. In general, due to the strong simplifications involved, past studies based on the Vlasov approach could only identify the order of magnitude of the instability threshold found by PIC simulations [13], but a quantitative agreement on the frequency of the instability and on its growth rate could not be shown.

In the following we will present a more general approach that addresses the highlighted limitations. As our focus is on proton accelerators, we will neglect the effect of radiation damping on the beam dynamics. The longitudinal focusing force from the rf system is assumed to be linear and, for simplicity, we will study the transverse motion and the stability of the bunch only in the horizontal plane, neglecting coupling to the vertical plane.

Examples and numerical tests in this article will refer to the realistic case of transverse instabilities observed at the LHC, which will be briefly introduced in Sec. II. In Sec. III we will introduce an effective way of characterizing both the dipolar and quadrupolar forces exerted by the e-cloud on the bunch distribution. In particular we will show that the dipolar forces of a realistic e-cloud can be accurately described by a small set of one-dimensional response functions and that the detuning introduced along the bunch by the e-cloud quadrupolar forces can be accurately modeled by a polynomial. In Sec. IV we will develop a method to solve the linearized Vlasov equation in the presence of an arbitrary detuning along the bunch and of a dipolar coherent force described by a set of response functions, as required to model the e-cloud. In Sec. V, after describing its implementation, the method will be applied to the realistic case of transverse instabilities observed at the LHC and will be benchmarked against macroparticle simulations performed with the same linearized model of the e-cloud. The results will also be compared against conventional simulations based on the PIC method, showing the capability of the proposed method to identify the frequency and the growth rate of the instability as well as the tune shift of the "rigid-bunch" mode. The impact of the transverse nonlinearities introduced by the e-cloud, which are neglected in the Vlasov treatment, will also be discussed.

Although the proposed method is developed to treat e-cloud effects, it can in fact be applied to different kinds of collective effects, in particular conventional and generalized wakefields, as will be briefly discussed in Appendix.

\section{CASE STUDY: INSTABILITIES DRIVEN BY E-CLOUD IN THE ARC QUADRUPOLES AT THE LHC}

In the examples and numerical tests discussed in the following we will refer to the realistic case of transverse instabilities observed at the LHC at injection energy, which are driven by the e-cloud in the superconducting quadrupoles of the collider. In spite of their modest length (they occupy only $7 \%$ of the accelerator length) the quadrupoles represent the main source of e-cloud instabilities in the LHC, due to the fact that the magnetic field gradient tends to favour the accumulation of electrons in the chamber (magnetic trapping [15]) and to concentrate a large electron density around the beam location, as shown by the snapshot of the distribution displayed in Fig. 1. The main parameters of the considered instability scenario are reported in Table I.

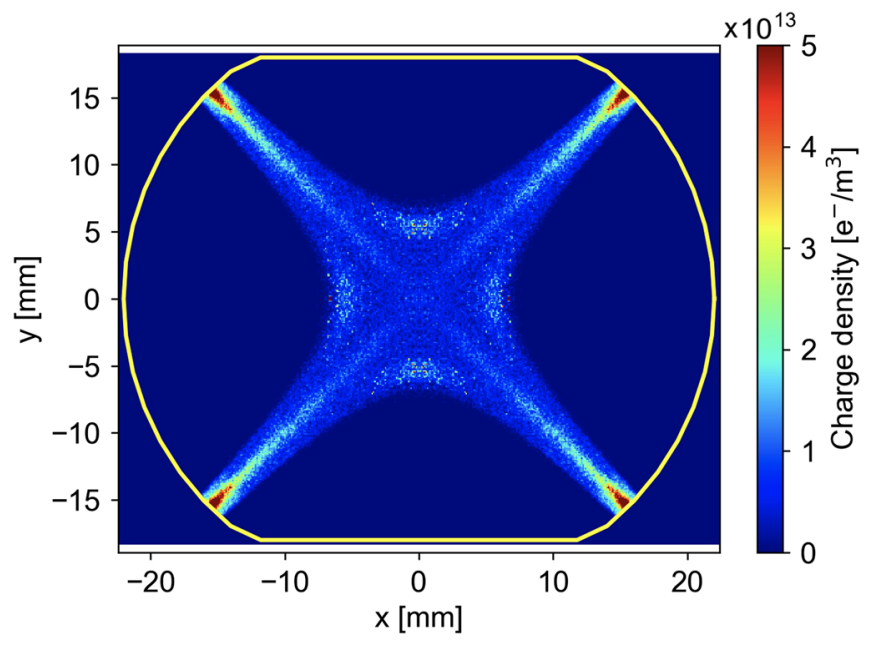

FIG. 1. Electron distribution in the LHC arc quadrupoles at injection energy as seen right before the arrival of a bunch (from a PIC simulation). 
TABLE I. Main parameters for the considered LHC scenario.
Beam energy [GeV]

Bunch population, $N_{b}$ [p/bunch]

R.m.s. bunch length, $\sigma_{b}[\mathrm{~cm}]$

R.m.s horizontal emittance (normalized) $[\mu \mathrm{m}]$

R.m.s vertival emittance (normalized) $[\mu \mathrm{m}]$

Ring circumference, $(2 \pi R)[\mathrm{km}]$

Horizontal beta function at the e-clouds, $\beta_{x}[\mathrm{~m}]$

Vertical beta function at the e-clouds, $\beta_{y}[\mathrm{~m}]$

Horizontal betatron tune, $Q_{x}$

Vertical betatron tune, $Q_{y}$

Synchrotron tune, $Q_{s}$

Slippage factor, $\eta$

Quadrupole gradient [T/m]

Fraction of the ring occupied by quadrupoles

$\mathrm{SEY}_{\max }$ at the quadrupole beam-screens
450

$1.2 \times 10^{11}$

9.7

2.5

2.5

26.7

92.7

93.2

62.27

60.295

$4.9 \times 10^{-3}$

$3.18 \times 10^{-4}$

12.1

$7 \%$

1.4
TABLE II. Numerical settings used in the macroparticle simulations.

\begin{tabular}{lc}
\hline \hline Number of e-cloud interactions & 8 \\
Number of macroparticles per e-cloud & $5 \times 10^{5}$ \\
Number of macroparticles per bunch & $5 \times 10^{6}$ \\
Number of longitudinal slices along the bunch & 200 \\
Transverse grid resolution at the beam location & $0.1 \mathrm{~mm}$ \\
\hline \hline
\end{tabular}

All macroparticle simulations mentioned in this work are performed with the PyECLOUD-PyHEADTAIL suite [8], using the numerical settings summarized in Table II, which followed from systematic convergence studies.

\section{LINEARIZED DESCRIPTION OF THE E-CLOUD FORCES}

In this section we introduce a method to build a linearized model of the forces exerted by the e-cloud on a circulating bunch. For this purpose we will use quick (single-pass) PIC simulations. In particular in Sec. III A we will characterize the tune modulation introduced by the e-cloud along the bunch and in Sec. III B we will address the dipolar forces exerted by the e-cloud in response to a distortion of the bunch distribution.

\section{A. Quadrupolar forces}

The presence of electrons in the beam chamber introduces focusing forces on the beam particles. In fact, during the bunch passage, the electrons are attracted toward the beam (the so-called "electron pinch" occurs) and the electron density at the beam location increases, as shown in the top part of Fig. 2 for the LHC case introduced in Sec. II ( $z$ defines the coordinate along the bunch, with $z>0$ corresponding to the head of the bunch). This effect results in a change of the focusing forces during the bunch passage and therefore in a betatron tune modulation with the
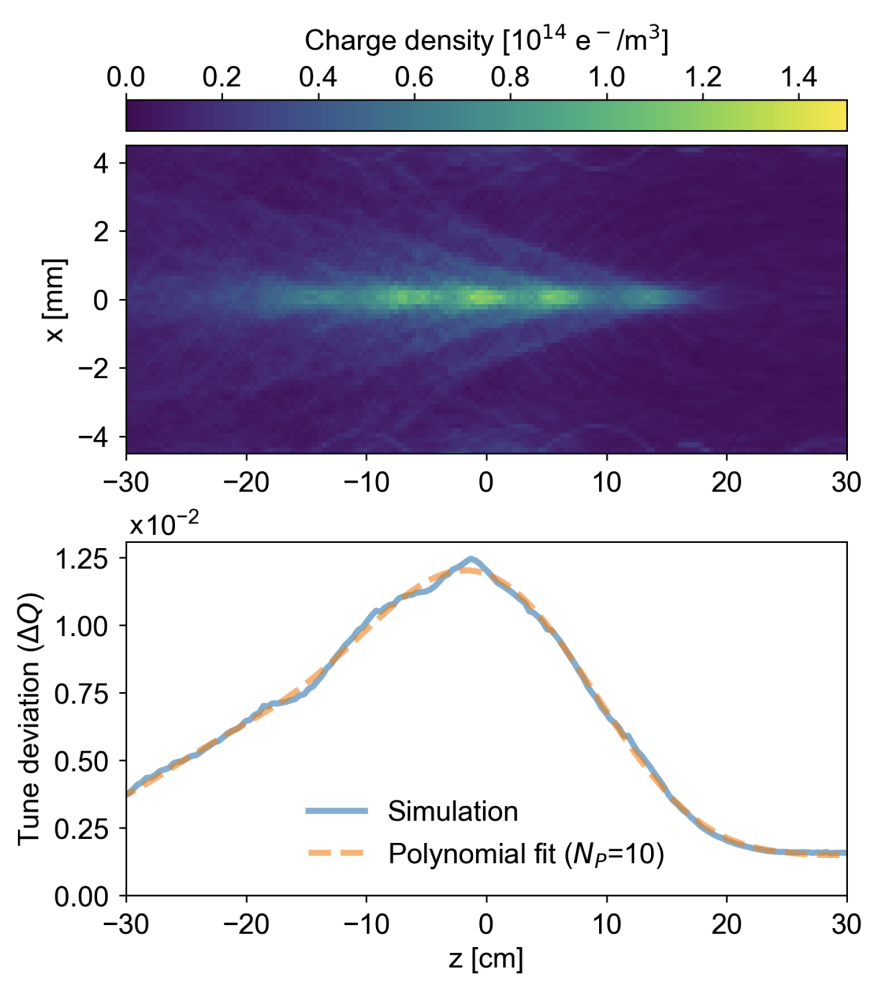

FIG. 2. Top: evolution of the electron density in the plane $y=0$ during the passage of a bunch. Bottom: corresponding effect on the particle betatron tune.

longitudinal coordinate. This is displayed in the bottom part of Fig. 2, where one can observe the tune shift increasing by about a factor of seven from the head to the core of the bunch and then slowly decaying toward the tail.

In the following the detuning along the bunch will be modeled using a polynomial:

$$
\Delta Q(z)=\sum_{n=0}^{N_{P}} A_{n} z^{n}
$$

Figure 2 shows that a realistic model can be obtained truncating the sum at $N_{P}=10$.

\section{B. Dipolar forces}

To describe the dipolar forces introduced by the e-cloud in response to distortions of the bunch shape, we consider a discrete set of functions $h_{n}(z)$ that satisfy the following orthogonality condition:

$$
\int h_{n}(z) h_{n^{\prime}}(z) d z=H_{n}^{2} \delta_{n, n^{\prime}}
$$

where $H_{n}$ is the norm of $h_{n}(z)$ and $\delta_{n, n^{\prime}}$ is the Kronecker delta.

The transverse centroid as a function of the position along the bunch, $\bar{x}(z)$, can be written as a linear combination of the $h_{n}$ functions: 

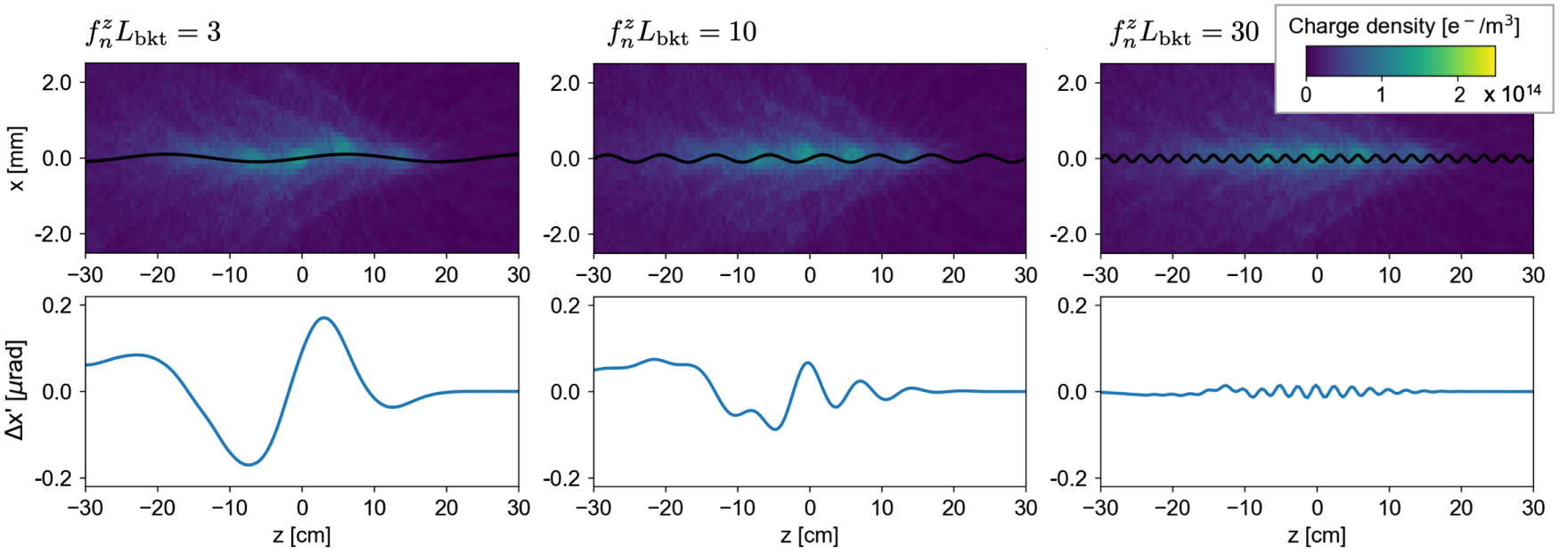

FIG. 3. Top: some of the applied test functions (in black) and corresponding evolution of the electron density. Bottom: dipolar kick measured along the bunch as an effect of the applied test function (the effect of the e-cloud quadrupolar forces is already subtracted).

$$
\bar{x}(z)=\sum_{n=0}^{\infty} a_{n} h_{n}(z)
$$

where the coefficients $a_{n}$ are given by the scalar product:

$$
a_{n}=\frac{1}{H_{n}^{2}} \int \bar{x}(z) h_{n}(z) d z \text {. }
$$

We call $k_{n}(z)$ the e-cloud kick along the bunch resulting from a distortion of the bunch distribution equal to $h_{n}(z)$. For sufficiently small amplitudes of the transverse distortion, the response of the e-cloud can be assumed to be linear and the resulting transverse kick along the bunch can be written using the superposition principle:

$\Delta x^{\prime}(z)=\sum_{n=0}^{\infty} a_{n} k_{n}(z)=\sum_{n=0}^{\infty} k_{n}(z) \int \bar{x}(\tilde{z}) \frac{h_{n}(\tilde{z})}{H_{n}^{2}} d \tilde{z}$.

Although the discussed method is valid for any set of orthogonal basis functions, for the practical examples presented in this work, we will choose $h_{n}(z)$ to be simple sinusoids in the form:

$$
h_{n}(z)=\left\{\begin{array}{ll}
\mathcal{A}_{n} \cos \left(2 \pi f_{n}^{z} z\right), & \text { if } n \text { is even } \\
\mathcal{A}_{n} \sin \left(2 \pi f_{n}^{z} z\right), & \text { if } n \text { is odd }
\end{array},\right.
$$

where

$$
f_{n}^{z}=\left\{\begin{array}{ll}
\frac{n}{2} \frac{1}{L_{\mathrm{bkt}}} & \text { if } n \text { is even } \\
\frac{n+1}{2} \frac{1}{L_{\mathrm{bkt}}} & \text { if } n \text { is odd }
\end{array},\right.
$$

$L_{\mathrm{bkt}}$ is the length of the rf bucket, and the amplitudes $\mathcal{A}_{n}$ are chosen to be equal to $0.1 \mathrm{~mm}$, corresponding to about $15 \%$ of the transverse r.m.s. beam size in the LHC case (see Table I).
The response functions $k_{n}(z)$ can be obtained through short (single-pass) PIC simulations, in which the distortion $h_{n}(z)$ is applied to the bunch and the corresponding kick is measured. It has been verified that, for similar or smaller amplitudes of the test functions, the response of the e-cloud is linear, i.e., the response function $k_{n}(z)$ scale linearly with the amplitude of the corresponding test function $h_{n}(z)$.

The detuning forces discussed in Sec. III A are subtracted from the measured kicks:

$$
k_{n}(z)=\Delta x_{\text {meas }}^{\prime}(z)-\frac{4 \pi}{\beta_{x}} \Delta Q(z) h_{n}(z)
$$

and will be treated separately.

Some of the measured response functions for our LHC test case are illustrated in Fig. 3. We observe that the strength of the resulting kick reduces for larger excitation frequency. This feature is a result of the inertia of the electrons, which prevents them from responding to very fast oscillations within the bunch. This is displayed in a more general way in Fig. 4 where the energy of all response functions (defined as $\int k_{n}^{2}(z) d z$ ) is displayed. For the considered LHC test case, the larger terms are observed at frequencies close to the electron oscillation frequency within the bunch [13]. Terms with $n>30$ have negligible energy, which means that the dipolar response of the e-cloud is fully described by the first 30 terms.

In Fig. 5 we test the capability of this approach to reconstruct the kick for a realistic distortion of the bunch shape obtained from an e-cloud instability simulated with the PIC method. A very good agreement with the PIC simulation is obtained even with a relatively small number of terms. This feature will result in a very reasonable computational burden for the calculation of the mode coupling matrix that will be presented in Sec. IV.

One can finally note that this description of the e-cloud dipolar forces with a small number of one-dimensional 


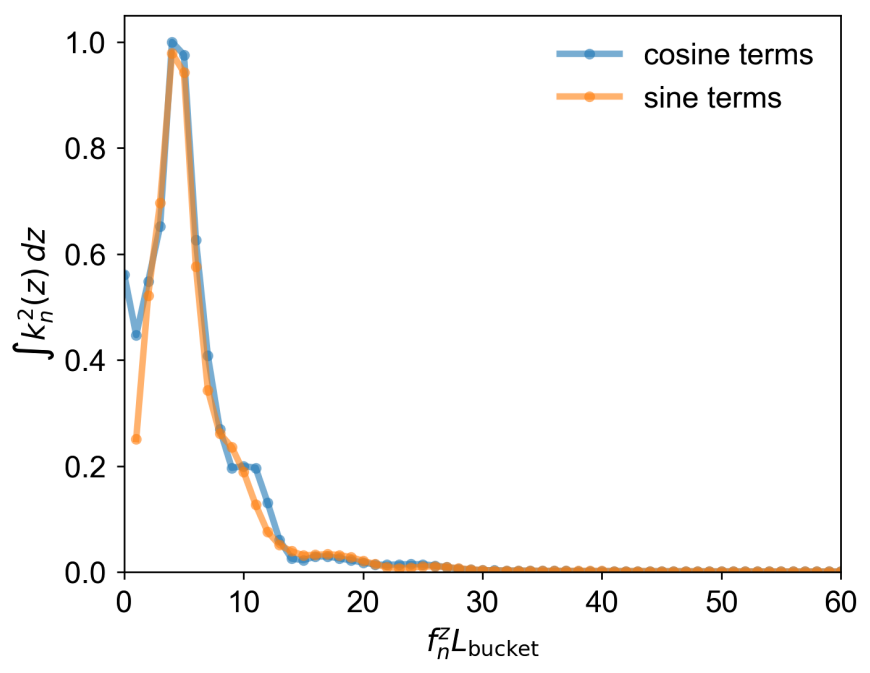

FIG. 4. Energy associated with the measured response functions $k_{n}(z)$. The curves are normalized to the maximum value.
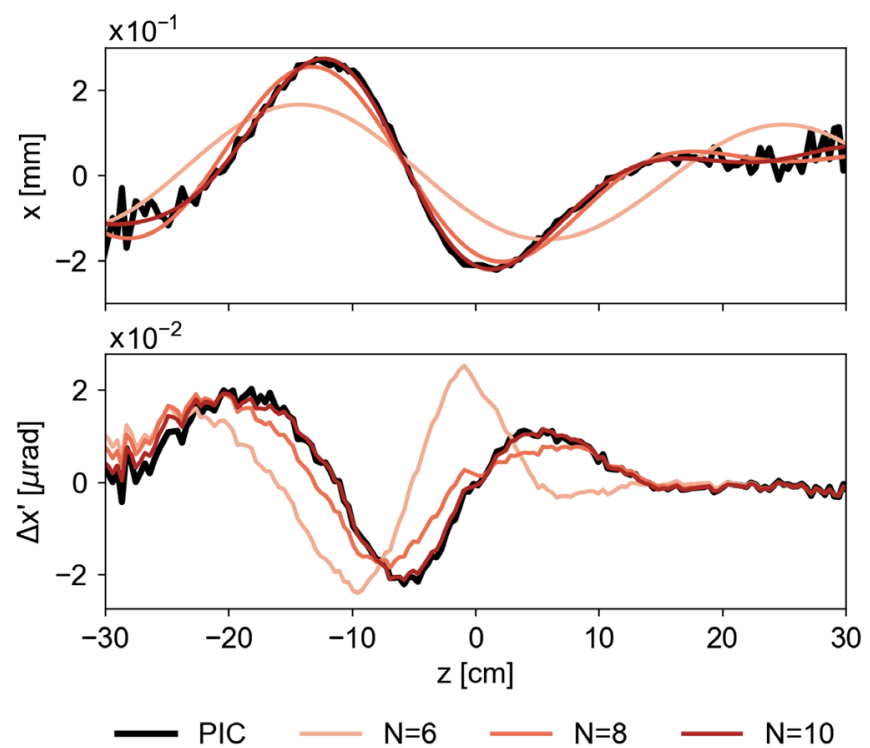

FIG. 5. Top: intrabunch oscillation as observed during an e-cloud instability simulated with the PIC method (black); the other traces show its reconstruction using Eq. (3) with an increasing number of terms. Bottom: transverse kick measured along the bunch in the same PIC simulations (black); the other traces show its reconstruction using Eq. (5).

functions, turns out to be much more compact than the twodimensional generalized wake function used in $[11,14]$.

\section{VLASOV TREATMENT}

To study the long-term stability of the bunch based on the linearized model introduced in Sec. III, we apply Vlasov equation [16] to the distribution function $\psi\left(x, x^{\prime}, z, \delta ; t\right)$, representing the phase space density of particles in the bunch. Considering only its first order perturbation $\Delta \psi$, our starting point is the so-called linearized Vlasov equation as expressed in Eq. (53) of Ref. [17], with an additional detuning term depending on the longitudinal phase space coordinates $\Delta Q(r, \phi)$, as done in Ref. [18]:

$$
\begin{gathered}
\frac{\partial \Delta \psi}{\partial t}-\omega_{0}\left(Q_{x 0}+\Delta Q(r, \phi)\right) \frac{\partial \Delta \psi}{\partial \theta_{x}}+\omega_{s} \frac{\partial \Delta \psi}{\partial \phi} \\
=-\frac{\eta g_{0}(r)}{\omega_{s} m_{0} \gamma} \frac{d f_{0}}{d J_{x}} \sqrt{\frac{2 J_{x} R}{Q_{x 0}}} \sin \theta_{x} F_{x}^{\mathrm{coh}}(z, t) .
\end{gathered}
$$

Here $F_{x}^{\mathrm{coh}}(z, t)$ is the transverse dipolar force due to the e-cloud; polar coordinates in the longitudinal phase space $(r, \phi)$ are defined such that:

$$
\begin{gathered}
z=r \cos \phi, \\
\delta=\frac{\omega_{s}}{v \eta} r \sin \phi ;
\end{gathered}
$$

polar coordinates in the transverse phase space $\left(J_{x}, \theta_{x}\right)$ are defined such that:

$$
\begin{gathered}
x=\sqrt{\frac{2 J_{x} R}{Q_{x}}} \cos \theta_{x}, \\
x^{\prime}=\sqrt{\frac{2 J_{x} Q_{x 0}}{R}} \sin \theta_{x} ;
\end{gathered}
$$

with $J_{x}$ being the horizontal action; $\omega_{0}$ is the revolution angular frequency; $Q_{x 0}$ is the unperturbed betatron tune; $\omega_{s}=\omega_{0} Q_{s}$ is the synchrotron angular frequency; $\eta$ is the slippage factor, $m_{0}$ is the particle mass, $v$ its velocity, $\gamma$ the corresponding relativistic factor, and $R=v / \omega_{0}$ is the accelerator radius. The unperturbed bunch distribution has been factorized as:

$$
\psi_{0}\left(J_{x}, r\right)=\frac{\eta v}{\omega_{s}} f_{0}\left(J_{x}\right) g_{0}(r)
$$

where the following normalization is chosen:

$$
\begin{gathered}
\int f_{0}\left(J_{x}\right) d J_{x}=\frac{N_{b}}{2 \pi}, \\
\int g_{0}(r) r d r=\frac{1}{2 \pi},
\end{gathered}
$$

$N_{b}$ being the number of particles in the bunch.

Generalizing the method discussed in Refs. $[9,17]$, we search for solutions in the form:

$$
\begin{aligned}
\Delta \psi\left(J_{x}, \theta_{x}, r, \phi, t\right)= & e^{j \Omega t} \sum_{p=-\infty}^{+\infty} f^{p}\left(J_{x}\right) e^{j p\left[\theta_{x}-\Delta \Phi(r, \phi)\right]} \\
& \times \sum_{l=-\infty}^{+\infty} R_{l}^{p}(r) e^{-j l \phi},
\end{aligned}
$$


where the complex frequency $\Omega$, the phase shift $\Delta \Phi(r, \phi)$ and the distribution functions $f^{p}\left(J_{x}\right)$ and $R_{l}^{p}(r)$ are to be found.

\section{A. Handling the detuning function}

Without loss of generality we can decompose the detuning term in two parts:

$$
\Delta Q(r, \phi)=\Delta Q_{R}(r)+\Delta Q_{\Phi}(r, \phi)
$$

where $\Delta Q_{R}(r)$ accounts for the transverse detuning with longitudinal amplitude and is defined as:

$$
\Delta Q_{R}(r)=\frac{1}{2 \pi} \int_{0}^{2 \pi} \Delta Q(r, \phi) d \phi .
$$

As a consequence, $\Delta Q_{\Phi}(r, \phi)$ introduces no average detuning over a synchrotron period

$$
\frac{1}{2 \pi} \int_{0}^{2 \pi} \Delta Q_{\Phi}(r, \phi) d \phi=0,
$$

but is only responsible for a phase shift as a function of the longitudinal coordinates.

Substituting Eqs. (17) and (18) into Eq. (9), we obtain:

$$
\begin{aligned}
& e^{j \Omega t} \sum_{p=-\infty}^{+\infty} f^{p}\left(J_{x}\right) e^{j p \theta_{x}} \sum_{l=-\infty}^{+\infty} R_{l}^{p}(r) e^{-j(p \Delta \Phi(r, \phi)+l \phi)} \\
& \times\left(j \Omega-j p \omega_{s} \frac{\partial \Delta \Phi}{\partial \phi}-j l \omega_{s}-j p \omega_{0}\left[Q_{x 0}+\Delta Q_{\Phi}+\Delta Q_{R}\right]\right) \\
& =-\frac{\eta g_{0}(r)}{\omega_{s} m_{0} \gamma} \frac{d f_{0}}{d J_{x}} \sqrt{\frac{2 J_{x} R}{Q_{x}}} \sin \theta_{x} F_{x}^{\mathrm{coh}}(z, t) .
\end{aligned}
$$

It is possible, without loss of generality, to choose the phase-shift function $\Delta \Phi$ to match the phase modulation induced by the term $\Delta Q_{\Phi}$ :

$$
\frac{\partial \Delta \Phi}{\partial \phi}=-\frac{\omega_{0}}{\omega_{s}} \Delta Q_{\Phi}(r, \phi) .
$$

Schenk et al. in Ref. [18] used an approach very similar to the one discussed so far, to handle the detuning introduced by nonlinear chromaticity. form:

Here we consider a detuning function in the general

$$
\Delta Q(z, \delta)=\sum_{n=0}^{N_{P}} A_{n} z^{n}+B_{n} \delta^{n},
$$

where the terms in $z^{n}$ allow modeling the detuning introduced by the e-cloud (as discussed in Sec. III A) and the terms in $\delta^{n}$ allow modelling linear and nonlinear chromaticity present in the machine. For a detuning function in this form, the following explicit expression of $\Delta \Phi$ can be found by integrating Eq. (22):

$$
\begin{aligned}
\Delta \Phi(r, \phi)= & -\frac{\omega_{0}}{\omega_{s}} \sum_{n=1}^{N_{P}} r^{n}\left[A_{n}\left(C_{n}(\phi)-\bar{C}_{n} \frac{\phi}{2 \pi}\right)\right. \\
& \left.+\left(\frac{\omega_{s}}{v \eta}\right)^{n} B_{n}\left(S_{n}(\phi)-\bar{S}_{n} \frac{\phi}{2 \pi}\right)\right],
\end{aligned}
$$

where the functions $C_{n}(\phi)$ and $S_{n}(\phi)$ are primitives of $\cos ^{n} \phi$ and $\sin ^{n} \phi$ respectively, which can be computed using the following recursive relations:

$$
\begin{gathered}
C_{0}(\phi)=\phi, \quad C_{1}(\phi)=\sin \phi, \\
C_{n}(\phi)=\frac{\cos ^{n-1} \phi \sin \phi}{n}+\frac{n-1}{n} C_{n-2}(\phi), \\
S_{0}(\phi)=\phi, \quad S_{1}(\phi)=-\cos \phi, \\
S_{n}(\phi)=-\frac{\sin ^{n-1} \phi \cos \phi}{n}+\frac{n-1}{n} S_{n-2}(\phi) .
\end{gathered}
$$

The constants $\bar{C}_{n}$ and $\bar{S}_{n}$ need to be chosen so that the condition in Eq. (20) is fulfilled:

$$
\begin{gathered}
\bar{C}_{n}=C_{n}(2 \pi)-C_{n}(0), \\
\bar{S}_{n}=S_{n}(2 \pi)-S_{n}(0) .
\end{gathered}
$$

Using Eqs. (18), (23), (22), and (24), it is possible to express $\Delta Q_{R}(r)$ as

$$
\Delta Q_{R}(r)=\sum_{n=0}^{N_{P}} r^{n}\left[A_{n} \frac{\bar{C}_{n}}{2 \pi}+B_{n}\left(\frac{\omega_{s}}{v \eta}\right)^{n} \frac{\bar{S}_{n}}{2 \pi}\right] .
$$

Having found a function $\Delta \Phi$ that satisfies Eq. (22) allows simplifying Eq. (21), obtaining:

$$
\begin{aligned}
& e^{j \Omega t} \sum_{p=-\infty}^{+\infty} f^{p}\left(J_{x}\right) e^{j p \theta_{x}} e^{-j p \Delta \Phi(r, \phi)} \\
& \times \sum_{l=-\infty}^{+\infty} R_{l}^{p}(r) e^{-j l \phi}\left(j \Omega-j p \omega_{0}\left(Q_{x 0}+\Delta Q_{R}(r)\right)-j l \omega_{s}\right) \\
& =-\frac{\eta g_{0}(r)}{\omega_{s} m_{0} \gamma} \frac{d f_{0}}{d J_{x}} \sqrt{\frac{2 J_{x} R}{Q_{x 0}}} \sin \theta_{x} F_{x}^{\operatorname{coh}}(z, t)
\end{aligned}
$$

As discussed in Refs. [17] and [19], it is possible to identify term by term the harmonics in $\theta_{x}$, showing that all terms with $|p| \neq 1$ vanish. Assuming that the transverse betatron tune is much larger than the synchrotron tune, we can neglect the fast-oscillation term $p=-1$, as discussed in Ref. [9]. This allows retaining only the term $p=1$, and leads to: 


$$
f^{1}\left(J_{x}\right) \propto \frac{d f_{0}}{d J_{x}} \sqrt{\frac{2 J_{x} R}{Q_{x 0}}}
$$

Therefore Eq. (17) becomes

$$
\begin{aligned}
& \Delta \psi\left(J_{x}, \theta_{x}, r, \phi, t\right) \\
& =e^{j \Omega t} e^{j \theta_{x}} \frac{d f_{0}}{d J_{x}} \sqrt{\frac{2 J_{x} R}{Q_{x}}} e^{-j \Delta \Phi(r, \phi)} \cdot \sum_{l=-\infty}^{+\infty} R_{l}(r) e^{-j l \phi}
\end{aligned}
$$

(where the proportionality constant in Eq. (33) is absorbed in the unknowns $R_{l}(r)$ ), and Eq. (32) simplifies into

$$
\begin{aligned}
& \sum_{l=-\infty}^{+\infty} R_{l}(r) e^{-j l \phi}\left(\Omega-Q_{x 0} \omega_{0}-\omega_{0} \Delta Q_{R}(r)-l \omega_{s}\right) \\
& =\frac{\eta g_{0}(r)}{2 \omega_{s} m_{0} \gamma} e^{-j \Omega t} e^{j \Delta \Phi(r, \phi)} F_{x}^{\mathrm{coh}}(z, t) .
\end{aligned}
$$

\section{B. Expressing the coherent force}

The dipolar force from the e-cloud can be expressed using Eq. (5), assuming that the force is distributed uniformly in the accelerator (smooth approximation):

$$
F_{x}^{\mathrm{coh}}(z, t)=\frac{m_{0} \gamma v^{2}}{2 \pi R} \sum_{n=0}^{N} k_{n}(z) \int \bar{x}(\tilde{z}, t) \frac{h_{n}(\tilde{z})}{H_{n}^{2}} d \tilde{z}
$$

where $\bar{x}(z, t)$ is the average position at the longitudinal position $z$, which can be written as

$$
\bar{x}(z, t)=\frac{1}{\lambda_{0}(z)} \iint d \tilde{x} d \tilde{x}^{\prime} \int d \tilde{\delta} \tilde{x} \Delta \psi\left(\tilde{x}, \tilde{x}^{\prime}, z, \tilde{\delta}, t\right),
$$

$\lambda_{0}(z)$ being the longitudinal bunch profile.

Substituting the expression of $\bar{x}(z, t)$ from Eq. (37) in Eq. (36) and using the expression of $\Delta \psi$ given by Eq. (34) we obtain:

$$
F_{x}^{\operatorname{coh}}(r, \phi, t)=-\frac{N_{b} m_{0} \gamma v \omega_{s}}{2 \pi \eta Q_{x 0}} e^{j \Omega t} \iint \tilde{r} d \tilde{r} d \tilde{\phi} e^{-j \Delta \Phi(\tilde{r}, \tilde{\phi})} \sum_{l^{\prime}=-\infty}^{+\infty} R_{l^{\prime}}(\tilde{r}) e^{-j l^{\prime} \tilde{\phi}} \sum_{n=0}^{N} k_{n}(r \cos \phi) \frac{h_{n}(\tilde{r} \cos \tilde{\phi})}{H_{n}^{2} \lambda_{0}(\tilde{r} \cos \tilde{\phi})},
$$

where all quantities have been expressed using polar coordinates, and where we have used the normalization condition from Eq. (15).

We can finally substitute the expression of the coherent force from Eq. (38) into Eq. (35), obtaining the following integral equation in the unknowns $\Omega$ and $R_{l}(r)$ :

$$
\begin{aligned}
& \sum_{l=-\infty}^{+\infty} R_{l}(r) e^{-j l \phi}\left(\Omega-Q_{x 0} \omega_{0}-\omega_{0} \Delta Q_{R}-l \omega_{s}\right) \\
& \quad=-\frac{N_{b} v}{4 \pi Q_{x 0}} e^{j \Delta \Phi(r, \phi)} g_{0}(r) \iint \tilde{r} d \tilde{r} d \tilde{\phi} e^{-j \Delta \Phi(\tilde{r}, \tilde{\phi})} \sum_{l^{\prime}=-\infty}^{+\infty} R_{l^{\prime}}(\tilde{r}) e^{-j l^{\prime} \tilde{\phi}} \sum_{n=0}^{N} k_{n}(r \cos \phi) \frac{h_{n}(\tilde{r} \cos \tilde{\phi})}{H_{n}^{2} \lambda_{0}(\tilde{r} \cos \tilde{\phi})}
\end{aligned}
$$

\section{Solving the equation}

Using the orthogonality property of the harmonic functions $e^{j l \phi}$ we can isolate an individual term of the sum on the 1.h.s. of Eq. (39):

$$
\begin{aligned}
R_{l}(r)\left(\Omega-Q_{x 0} \omega_{0}-\omega_{0} \Delta Q_{R}-l \omega_{s}\right)= & -\frac{N_{b} v}{8 \pi^{2} Q_{x 0}} g_{0}(r) \int_{0}^{2 \pi} d \phi e^{j l \phi} e^{j \Delta \Phi(r, \phi)} \iint \tilde{r} d \tilde{r} d \tilde{\phi} e^{-j \Delta \Phi(\tilde{r}, \tilde{\phi})} \\
& \times \sum_{l^{\prime}=-\infty}^{+\infty} R_{l^{\prime}}(\tilde{r}) e^{-j l^{\prime} \tilde{\phi}} \sum_{n=0}^{N} k_{n}(r \cos \phi) \frac{h_{n}(\tilde{r} \cos \tilde{\phi})}{H_{n}^{2} \lambda_{0}(\tilde{r} \cos \tilde{\phi})} .
\end{aligned}
$$

Following the standard approach described in Refs. $[10,14,20]$, we expand the radial functions $R_{l}(r)$ using orthogonal functions:

$$
R_{l}(r)=W_{l}(r) \sum_{m=0}^{+\infty} b_{l m} f_{l m}(r)
$$

where $W_{l}(r)$ is an arbitrary function, which can be chosen to improve the convergence properties, and the functions $f_{l m}(r)$ need to satisfy the orthogonality condition:

$$
\int f_{l m}(r) f_{l m^{\prime}}(r) w_{l}(r) d r=F_{l m} \delta_{m, m^{\prime}}
$$

$w_{l}(r)$ being a suitable weight function. 
We substitute in Eq. (40) the expansion from Eq. (41) and we apply to both sides the integral $\frac{1}{F_{l m}} \int d r w_{l}(r) f_{l m}(r) \frac{(*)}{W_{l}(r)}$, obtaining:

$$
\begin{aligned}
b_{l m}(\Omega & \left.-Q_{x 0} \omega_{0}-l \omega_{s}\right)-\frac{\omega_{0}}{F_{l m}} \sum_{m^{\prime}=0}^{+\infty} b_{l m^{\prime}} \int d r w_{l}(r) \Delta Q_{R}(r) f_{l m}(r) f_{l m^{\prime}}(r) \\
= & -\frac{N_{b} v}{8 \pi^{2} Q_{x 0} F_{l m}} \sum_{l^{\prime} m^{\prime}} b_{l^{\prime} m^{\prime}} \sum_{n=0}^{N} \iint d r d \phi e^{j l \phi} e^{j \Delta \Phi(r, \phi)} w_{l}(r) f_{l m}(r) \frac{g_{0}(r)}{W_{l}(r)} k_{n}(r \cos \phi) \\
& \times \iint \tilde{r} d \tilde{r} d \tilde{\phi} e^{-j l^{\prime} \tilde{\phi}} e^{-j \Delta \Phi(\tilde{r}, \tilde{\phi})} f_{l^{\prime} m^{\prime}}(\tilde{r}) \frac{W_{l^{\prime}}(\tilde{r})}{\lambda_{0}(\tilde{r} \cos \tilde{\phi})} \frac{h_{n}(\tilde{r} \cos \tilde{\phi})}{H_{n}^{2}} .
\end{aligned}
$$

By introducing the matrices

$$
\begin{aligned}
M_{l m, l^{\prime} m^{\prime}}= & -\frac{N_{b} v}{8 \pi^{2} Q_{x 0} F_{l m}} \sum_{n=0}^{N} \iint d r d \phi e^{j l \phi} e^{j \Delta \Phi(r, \phi)} w_{l}(r) f_{l m}(r) \frac{g_{0}(r)}{W_{l}(r)} k_{n}(r \cos \phi) \\
& \times \iint \tilde{r} d \tilde{r} d \tilde{\phi} e^{-j l^{\prime} \tilde{\phi}} e^{-j \Delta \Phi(\tilde{r}, \tilde{\phi})} f_{l^{\prime} m^{\prime}}(\tilde{r}) \frac{W_{l^{\prime}}(\tilde{r}) h_{n}(\tilde{r} \cos \tilde{\phi})}{\lambda_{0}(\tilde{r} \cos \tilde{\phi}) H_{n}^{2}}
\end{aligned}
$$

and

$$
\tilde{M}_{l m, l^{\prime} m^{\prime}}=\delta_{l, l^{\prime}} \frac{\omega_{0}}{F_{l m}} \int d r w_{l}(r) \Delta Q_{R}(r) f_{l m}(r) f_{l m^{\prime}}(r),
$$

we can rewrite Eq. (43) in the more compact form:

$$
b_{l m}\left(\Omega-Q_{x 0} \omega_{0}-l \omega_{s}\right)=\sum_{l^{\prime} m^{\prime}}\left(M_{l m, l^{\prime} m^{\prime}}+\tilde{M}_{l m, l^{\prime} m^{\prime}}\right) b_{l^{\prime} m^{\prime}}
$$

In Eq. (46), we can recognize the structure of an eigenvalue problem, where the eigenvalues provide the complex frequencies $\Omega$ of the bunch eigenmodes, and the eigenvectors $b_{l m}$ define the corresponding phase space distribution through Eqs. (34) and (41).

\section{Gaussian bunch}

For the practical case of a bunch having a Gaussian longitudinal distribution:

$$
g_{0}(r)=\frac{1}{2 \pi \sigma_{b}^{2}} e^{-\frac{r^{2}}{2 \sigma_{b}^{2}}}
$$

(where $\sigma_{b}$ is the r.m.s. bunch length) it is convenient to use the generalized Laguerre polynomials [21] to express the radial dependence of the eigenmodes:

$$
f_{l m}(r)=L_{m}^{|l|}\left(a r^{2}\right)
$$

as done in $[10,14,22]$, where we choose $a=0.5 \sigma_{b}^{-2}$. For these functions to satisfy the orthogonality condition in Eq. (42), the suitable weight function $w_{l}(z)$ is

$$
w_{l}(r)=2 a r e^{-a r^{2}}\left(a r^{2}\right)^{|l|}
$$

and the coefficients $F_{l m}$ are

$$
F_{l m}=\frac{(|l|+m) !}{m !} .
$$

Following Refs. [10,14] we choose the shape function:

$$
W_{l}(r)=\left(\frac{r}{r_{b}}\right)^{|l|} e^{-a r^{2}}
$$

with $r_{b}=4 \sigma_{b}$.

With these choices, when the phase-shift term is negligible, the eigenvector corresponding to a rigid bunch oscillation has the form:

$$
b_{l m}=b_{00} \delta_{l} \delta_{m},
$$

and the corresponding complex tune shift can be found substituting Eq. (52) into Eq. (46) obtaining:

$$
\frac{\bar{\Omega}}{\omega_{0}}-Q_{x 0}=\frac{M_{00,00}+\tilde{M}_{00,00}}{\omega_{0}} .
$$

We can notice that the tune shift is the sum of two terms, the first one due to dipolar forces $\left(M_{00,00} / \omega_{0}\right)$ and the second one due to quadrupolar forces $\left(\tilde{M}_{00,00} / \omega_{0}\right)$.

\section{IMPLEMENTATION, NUMERICAL EXPERIMENTS AND BENCHMARKS}

The implementation of the method described above essentially consists in the calculation of the matrices $M_{l m, l^{\prime} m^{\prime}}$ and $\tilde{M}_{l m, l^{\prime} m^{\prime}}$ and in the solution of the eigenvalue problem in Eq. (46). 
The computation of the matrix $M_{l m, l^{\prime} m^{\prime}}$ from Eq. (44) can be optimized using the fact that the matrix is in the form:

$$
M_{l m, l^{\prime} m^{\prime}}=\sum_{n=0}^{N} R_{l m n} \tilde{R}_{l^{\prime} m^{\prime} n}
$$

The terms $R_{l m n}$ and $\tilde{R}_{l^{\prime} m^{\prime} n}$ are calculated using the trapezoidal method to compute all the involved integrals. They are stored in memory to be reused multiple times in the computation of different elements of the matrix $M_{l m, l^{\prime} m^{\prime}}$.

The computation of the matrix $\tilde{M}_{l m, l^{\prime} m^{\prime}}$ is less heavy, due to the fact that several of its elements are zeros and that only a single-dimension integration is required. Once the matrices are computed the eigenvalue problem can be solved using a standard linear-algebra package.

\section{A. Application to $\mathrm{LHC}$ case}

We applied the method described in Sec. IV to the case of the LHC instabilities presented in Sec. II.

The bunch stability is studied as a function of the "e-cloud strength," defined as a scaling factor that is applied on all the e-cloud forces acting on the beam. Such a quantity can represent the fraction of the accelerator length in which the e-cloud is developing or, in first approximation, the electron density. In the results presented in this section, an e-cloud strength equal to one corresponds to the e-cloud parameters reported in Table I.

The e-cloud dipolar forces, the intra-bunch phase shift due to the term $\Delta Q_{\Phi}$, and the detuning with longitudinal amplitude $\Delta Q_{R}$ are introduced in steps, to identify the effect due to each of them. Based on convergence checks, in all calculations based on the Vlasov method we considered terms up to $|l|=7$ and $m=40$.

Figure 6(a) shows the tune shifts of the eigenmodes (obtained from the real part of the computed eigenvalues $\Omega$ ) for the case in which only the e-cloud dipolar forces are considered, while the quadrupolar effects are not included $\left(\Delta Q_{\Phi}=\Delta Q_{R}=0\right)$. The color code represents the growth rate of the eigenmode, as obtained from the imaginary part of $\Omega$, while the orange dashed line shows the "rigid-bunch" tune shift estimated from Eq. (53).

The tune deviation of the strongest unstable modes with respect to the unperturbed tune is observed to be negative. The bunch is essentially stable for an e-cloud strength below 0.8 , while for larger strength instabilities are triggered by mode coupling. The instability threshold is visible also in the blue continuous line in Fig. 9, showing the growth rate for the most unstable mode as computed by the Vlasov method.

In Fig. 7(a) we show the results of computations obtained by introducing the phase shift from the e-cloud detuning $\left(\Delta Q_{\Phi} \neq 0\right)$ while we keep no detuning with longitudinal amplitude $\left(\Delta Q_{R}=0\right)$. We observe that, while the tune shift for most of the modes is similar to the case with no quadrupolar forces, the coupling-decoupling behavior of the modes is affected by the phase-shift. This results in a faster instability, as visible from the continuous orange line in Fig. 9.

The effect of introducing also the term responsible for the e-cloud detuning with longitudinal amplitude $\left(\Delta Q_{R} \neq 0\right)$ is much more visible, as shown in Fig. 8(a). Modes with different radial distributions receive different positive tune shifts from the quadrupolar forces. This generates characteristic "fans of modes" (indicated by red arrows in Fig. 8) associated to each synchrotron sideband. The negative tune shift introduced by the dipolar forces on the "rigid-bunch" eigenmode is fully canceled by the quadrupolar effect. In fact, as a net effect of the two forces, it becomes slightly positive, as shown by the orange dashed line in Fig. 8(a). Also, the mode coupling behavior changes with respect to Fig. 7(a) and a larger number of unstable lines is observed. The growth rate for the most unstable mode as a function of the e-cloud strength is shown by the green line in Fig. 9. For strengths below 1.0, a slight increase in the growth rate is observed with respect to the case with $\Delta Q_{\Phi} \neq 0$ and $\Delta Q_{R}=0$, while a decrease of the growth rate is observed for higher strengths.

\section{B. Benchmark against macroparticle simulations using the linearized e-cloud model}

In order to validate the method described in Sec. IV and the corresponding implementation, the bunch dynamics in the configurations described in Sec. VA has been simulated with macroparticles using the PyHEADTAIL code, applying the same linearized model for the e-cloud dipolar and quadrupolar forces.

This simulation method is in itself an interesting alternative to the PIC simulations, as it has a much reduced computational burden (for the tests presented in this work we experienced a gain in computational time of more than one order of magnitude) and allows easily including effects that are difficult to model with the Vlasov approach, e.g., external transverse nonlinearities, complex feedback systems, or nonlinear longitudinal motion.

To compare against the eigenmode tune shifts found with the Vlasov method, we use the Sussix algorithm [23] to identify the different spectral components on the simulated bunch motion. In order to be able to detect also lines that would not be visible on the motion of the bunch centroid, we use the information on the transverse position along the bunch, which is recorded at each turn by the simulator. For this purpose, we apply the Sussix algorithm to the following auxiliary quantity computed at each turn $n$ : 
(a)

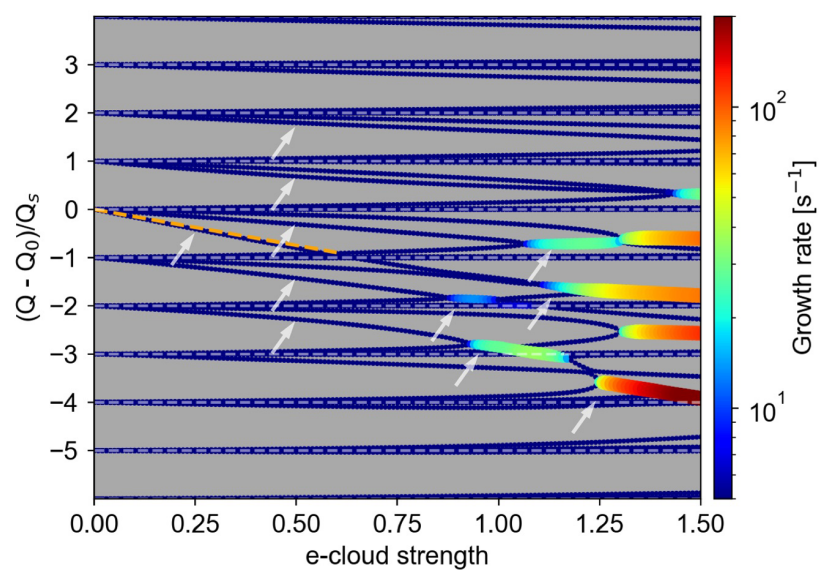

(b)

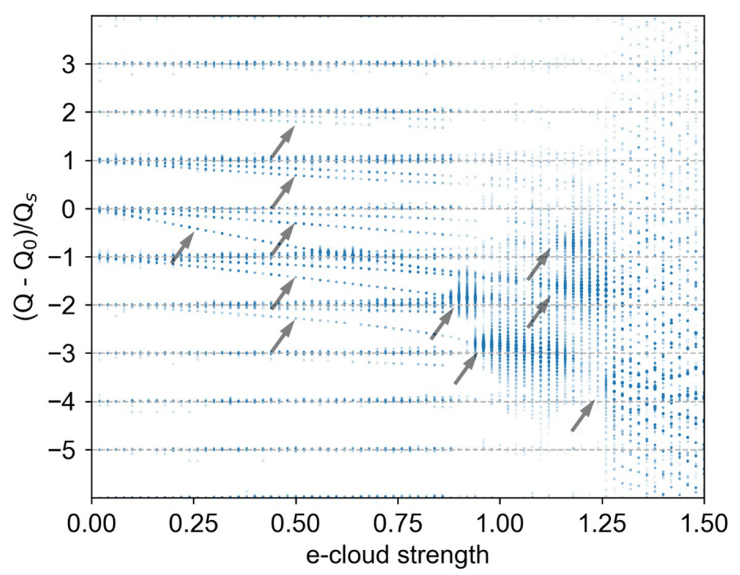

FIG. 6. (a) Frequencies of the eigenmodes computed with the Vlasov method for the case $\Delta Q_{R}=\Delta Q_{\Phi}=0$ (the color code is proportional to the mode's growth rate). (b) Spectrum of the transverse motion for the same configuration, computed using PyHEADTAIL macroparticle simulations with the linearized e-cloud model. The arrows in the plots highlight distinctive features (behavior of certain modes, observation of mode coupling) that are visible with both methods.

(a)

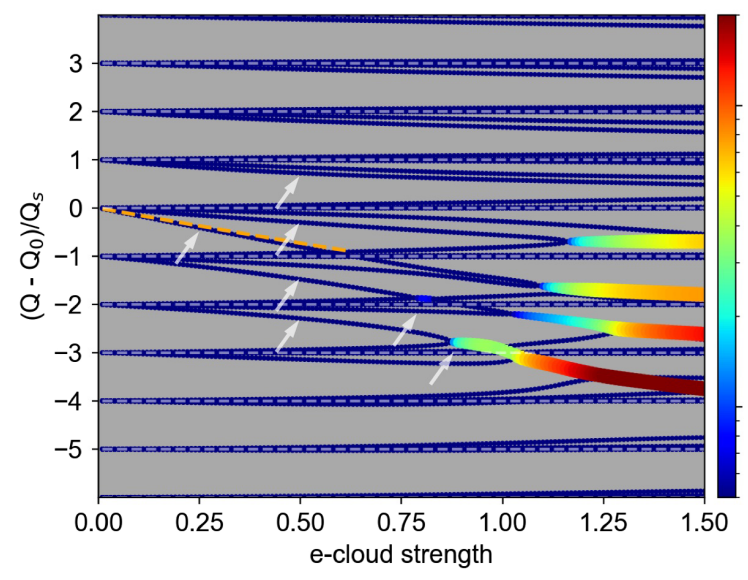

(b)

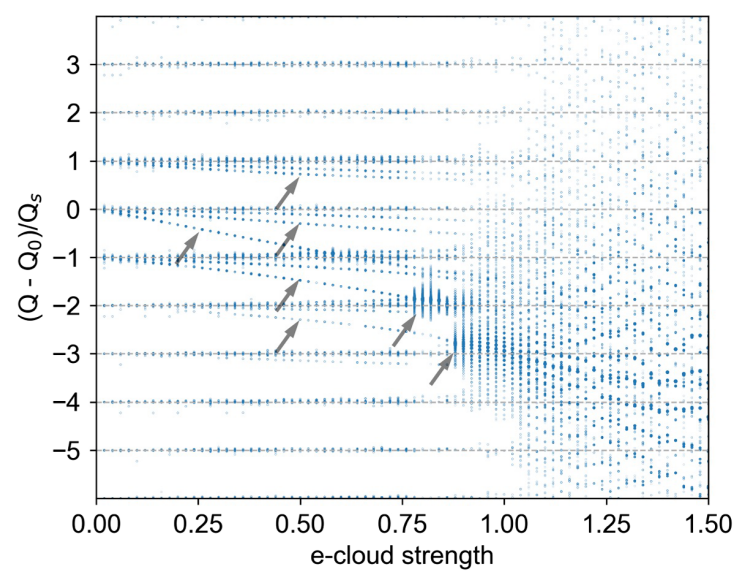

FIG. 7. Same as Fig. 6 for the case $\Delta Q_{R}=0, \Delta Q_{\Phi} \neq 0$. (a) Eigenvalues from linearized Vlasov equation, (b) PyHEADTAIL simulations (linearized e-could model).

(a)

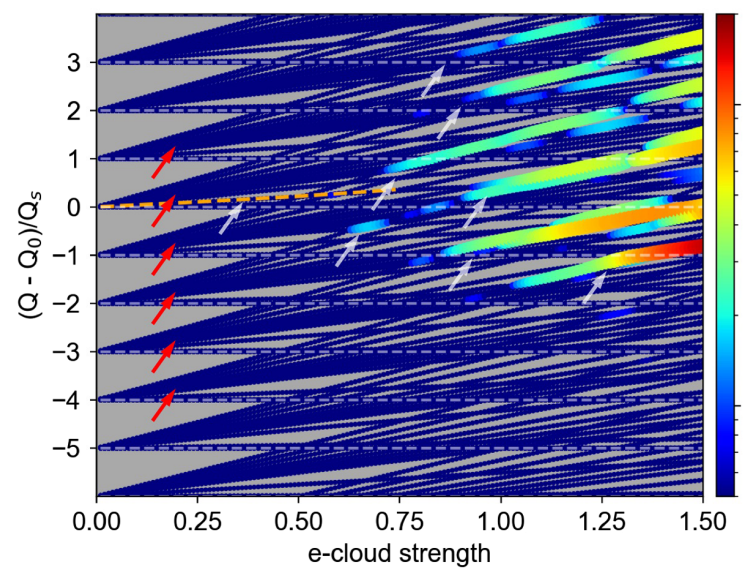

(b)

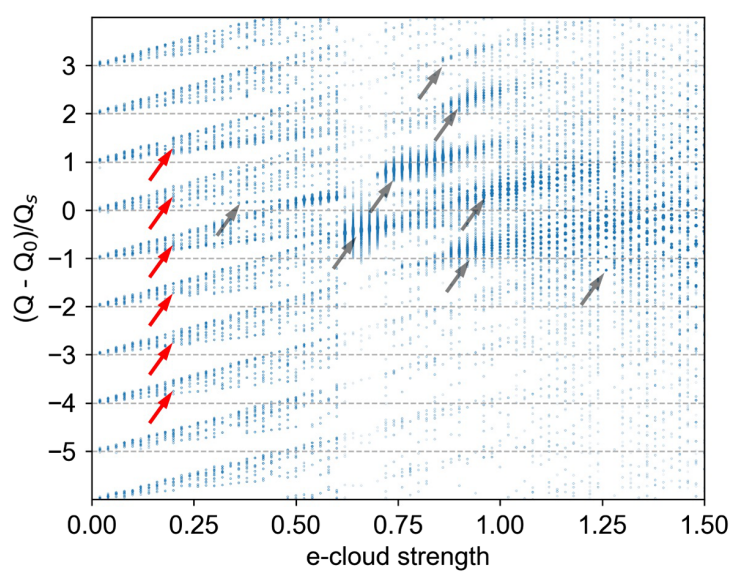

FIG. 8. Same as Fig. 6 for the case $\Delta Q_{R} \neq 0, \Delta Q_{\Phi} \neq 0$. (a) Eigenvalues from linearized Vlasov equation, (b) PyHEADTAIL simulations (linearized e-could model). 


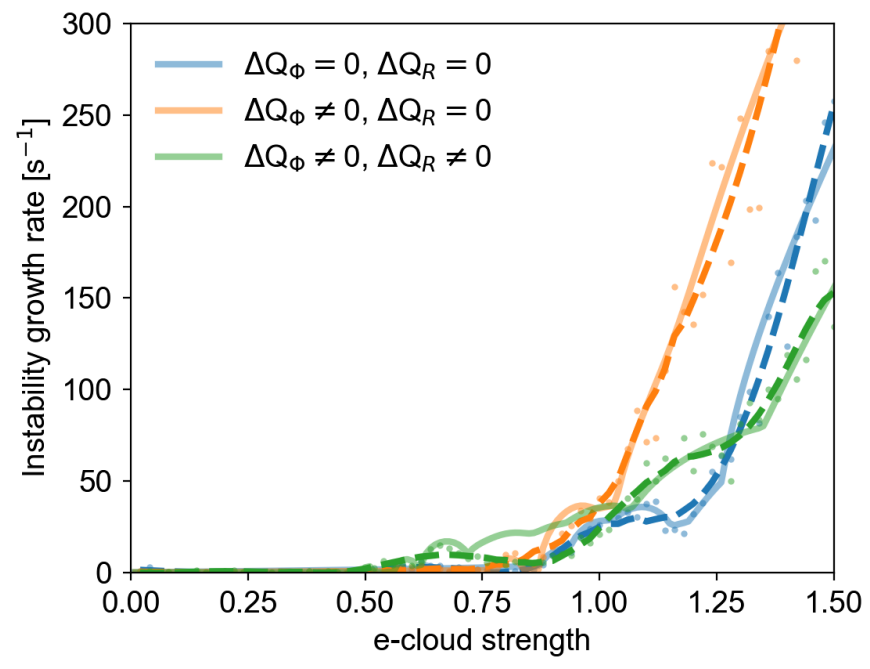

FIG. 9. Instability growth rate estimated with the Vlasov method (continuous lines) and with macroparticle simulations using the linearized model (dots). The dashed lines are a smoothed version of the data represented by the dots.

$$
X_{n}=\sum_{k=0}^{N_{k}} \int_{-\frac{L_{\mathrm{bkt}}}{2}}^{\frac{L_{\mathrm{bkt}}}{\mathrm{k}}} \bar{x}_{n}(z) e^{j 2 \pi k z / L_{\mathrm{bkt}}} d z
$$

which is built by summing projections of the intrabunch position over sinusoids of increasing frequency (a similar approach has been used to study transverse mode coupling driven by impedances in [24]). In the considered cases, terms of the sum with $k>10$ are found to be negligible.

(a)

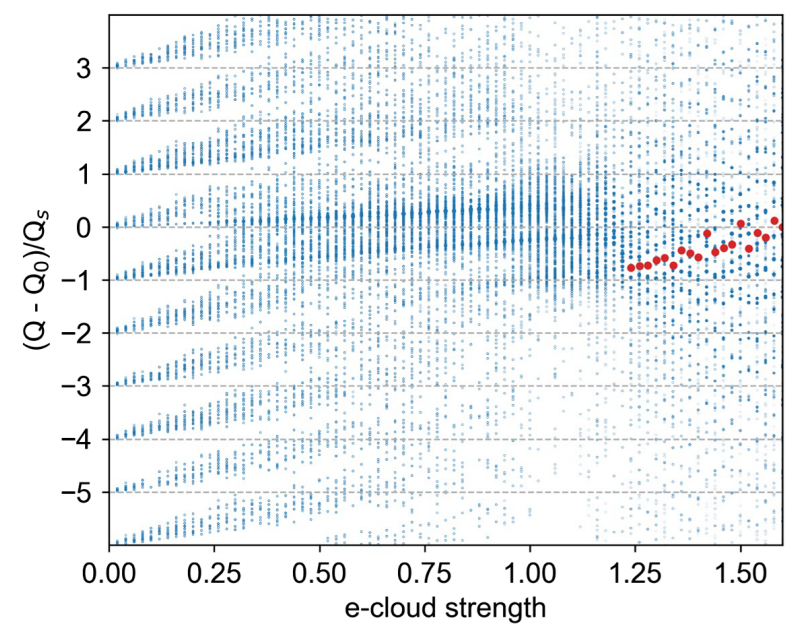

The resulting spectra are shown in Figs. 6(b), 7(b), and 8(b), respectively for the case with no quadrupolar forces, with the phase-shift term alone and with the full quadrupolar forces. It can be noticed that, for large e-cloud strengths the instability becomes very fast, making the spectral analysis less accurate. Very good agreement is found when comparing these spectra against the eigenmode frequencies predicted by the Vlasov method, which are displayed in Figs. 6(a), 7(a), and 8(a). The arrows in the plots highlight distinctive features (behavior of certain modes, observation of mode coupling) that are visible using both methods.

The instability growth rate has been estimated from the PyHEADTAIL simulations by applying an exponential fit on the recorded centroid motion. The result of the fits in the different cases are shown by the dots in Fig. 9. The dashed lines are obtained by applying a standard smoothing algorithm (Savitzky-Golay [25]) to remove the noise introduced by the fit uncertainty and by fluctuations in the initial macroparticle distribution. We can observe that indeed the dashed lines obtained in this way from the macroparticle simulations agree well with the continuous lines obtained with the Vlasov method.

\section{Comparison against particle-in-cell simulations and effect of transverse non-linearities}

In the Sec. V B we have compared the results of the Vlasov method against macroparticle simulations implementing our linearized description of the e-cloud.

To check whether the linearized description is indeed appropriate to describe the behavior of a realistic e-cloud, we now compare the same results against conventional

(b)

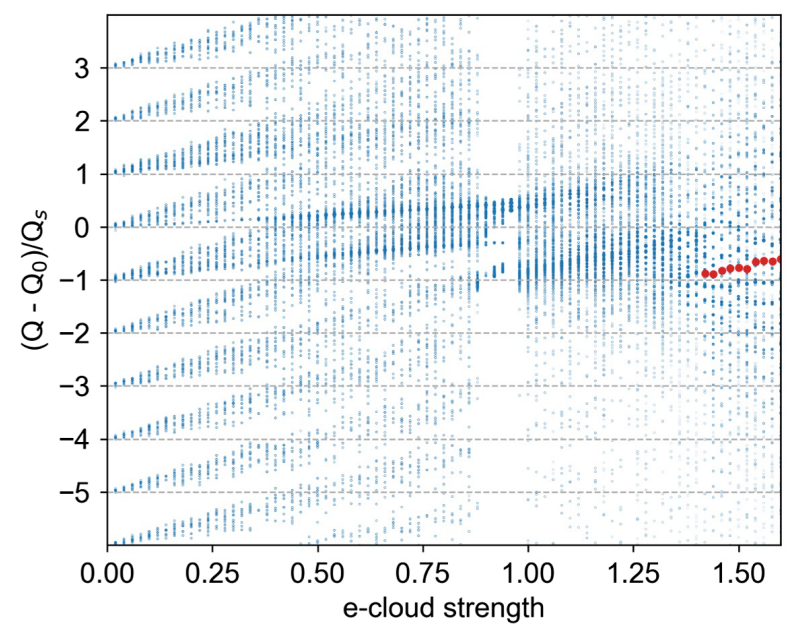

FIG. 10. (a) Spectrum of the transverse motion obtained from PyHEADTAIL macroparticle simulations performed with the PIC method. (b) Spectrum of the transverse motion obtained from macroparticle simulations performed with the linearized model and a static nonlinear map to model the e-cloud transverse nonlinearities. In the range in which the instability is very fast, the strongest spectral component is highlighted in red. 


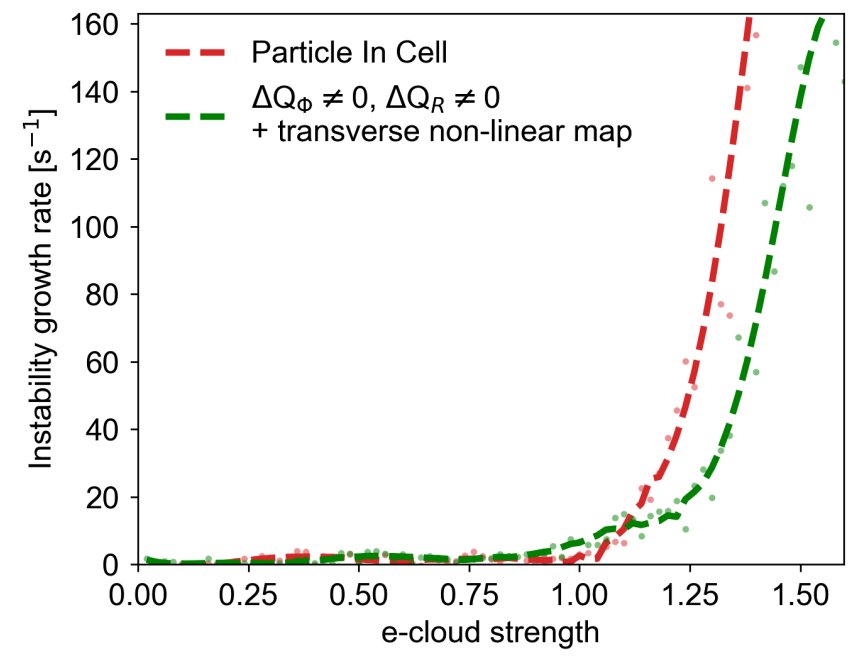

FIG. 11. Dots: Instability growth rate obtained by macroparticle simulations with the PIC method (in red), and with the linearized model and a static non-linear map to model the e-cloud transverse nonlinearities (in green). Dashed lines: smoothed version of the data represented by the dots.

"brute-force" simulations in which the full dynamics of the e-cloud is simulated by macroparticles, and the PIC method is used to compute the forces generated by the beam and by the e-cloud. In these simulations the nonlinearities of the e-cloud field with respect to the transverse position are also present, while this aspect is neglected by the linearized model used for all results shown in Secs. VA and V B.

For comparison, we apply to the bunch motion from the PIC simulations the frequency analysis method introduced in Sec. V B. The resulting spectrum as a function of the e-cloud strength is shown in Fig. 10(a) and the observed instability growth rate is shown by the red curve in Fig. 11.

In spite of the mentioned approximations, several key features are correctly predicted by the Vlasov method: (i) An excellent agreement is observed on the tune shift for the rigid-bunch mode, as shown in Fig. 12. The tune shift expected from the Vlasov method shown by the blue line is estimated using Eq. (53), while for the simulation the tune is obtained applying the Sussix algorithm on the recorded bunch-centroid position. The orange and green lines in Fig. 12 show the tune shift that would be expected with dipolar forces alone (obtained by imposing $\Delta Q(r, \phi)=0$ and therefore $\tilde{M}_{l m, l^{\prime} m^{\prime}}=0$ ) and with quadrupolar forces alone (obtained by imposing $k_{n}(z)=0$ and therefore $\left.M_{l m, l^{\prime} m^{\prime}}=0\right)$. It is evident that both forces from the e-cloud need to be taken into account in order to correctly predict the tune shift. (ii) The frequency spectrum from the PIC simulations [Fig. 10(a)] confirms the existence of "fans of modes" associated to each synchrotron sideband, which, as discussed in Sec. V B, are a direct result of the e-cloud quadrupolar forces. (iii) The strong instability observed in the PIC simulations for e-cloud strengths above 1.25 has a frequency in the range $-1<\left(Q-Q_{0}\right) / Q_{s}<0$, which is

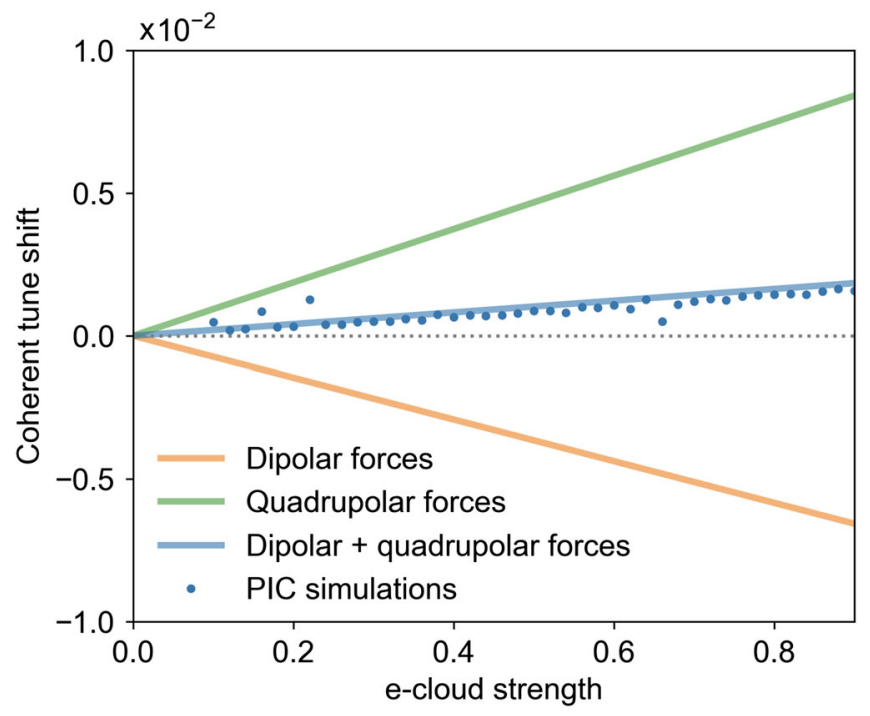

FIG. 12. Tune shift of the "rigid-bunch" mode estimated with the Vlasov approach (continuous lines) and obtained by macroparticle simulations performed with the PIC method.

very similar to that of the most unstable modes identified by the Vlasov method in Fig. 8. We underline that including the quadrupolar forces from the e-cloud was fundamental to correctly predict this feature. (iv) The instability growth rate observed in the PIC simulation for e-cloud strengths above 1.25 is similar to that of the most unstable mode identified by the Vlasov method, as it can be observed comparing the Fig. 11 (red line) and Fig. 9 (green line).

The main difference observed between the PIC simulations and the results from the Vlasov method concerns other unstable modes which are found by the Vlasov method in the range $0<\left(Q-Q_{0}\right) / Q_{s}<4$. These unstable modes are also visible in the macroparcoparticle simulations performed with the linearized model [Fig. 8(b)] but not in the spectra from the PIC simulations [Fig. 10(a)].

Our hypothesis is that those modes are damped by the e-cloud transverse non-linearities (such a stabilizing effect of the e-cloud is also mentioned in [26]). We used macroparticle simulations with the e-cloud linearized model to verify this assumption. For this purpose, we added to the linearized simulation model with dipolar and quadrupolar forces [the one used to produce the results in Fig. 8(b)] a static non-linear map independent on the $z$ coordinate. The latter is obtained from the field map of the simulated electron pinch after removing the linear part (already included in the linearized model), and averaging along $z$. The transverse spectrum obtained by these simulations is shown in Fig. 10(b). Comparing Fig. 10(b) against Fig. 8(b) confirms that the introduction of the transverse nonlinearities strongly suppresses the spectral lines in the range $0<\left(Q-Q_{0}\right) / Q_{s}<4$ resulting in a spectrum very similar to the one obtained by the PIC simulations [Fig. 10(a)]. Furthermore, the instability 
growth rate is found to be similar, as it can be observed by comparing the two curves in Fig. 11.

\section{SUMMARY AND CONCLUSIONS}

We have introduced a linearized model to describe dipolar and quadrupolar forces from an e-cloud. A polynomial has been used to describe the detuning forces along the bunch, and a small set of one-dimensional response functions has been used to describe the dipolar forces resulting from a transverse distortion of the bunch distribution.

Using a realistic LHC case, we have shown that our linearized model is capable of reproducing the effect on the bunch at the level of a single turn.

We have then developed a Vlasov method to study the effect of the linearized e-cloud on the long-term stability of the bunch. In this framework, it has been found convenient to decompose the detuning along the bunch in two terms, one responsible for a phase-shift and the other responsible for detuning with longitudinal amplitude. We have shown that both these terms play a relevant role in defining the spectral properties of the bunch motion, as well as the growth rate of the unstable modes.

The proposed Vlasov method has been benchmarked using macroparticle simulations based on the linearized model and the results have been compared against conventional "brute-force" simulations, in which the coupled dynamics of the bunch and the e-cloud is computed using the PIC method. The proposed approach is found to correctly predict different features observed in the PIC simulations: the tune shift for the rigid bunch mode agrees very well with the prediction from the Vlasov method; the transverse spectrum obtained by the PIC simulation shows the "fans of modes" that are predicted by the Vlasov method as a result of the e-cloud quadrupolar forces; the instabilities observed in the PIC simulations have frequencies and rise times that are very similar to those of the strongest mode identified by the Vlasov method.

Weaker unstable modes that are predicted by the Vlasov solver and shown by macroparticle simulations with the linearized model are not observed in the PIC simulation. These modes are in fact stabilized by the e-cloud non-linearities, as has been shown using macroparticle simulations with the linearized model and adding a static nonlinear map as a simplified model of the cloud nonlinearities.

\section{ACKNOWLEDGMENTS}

We are very grateful to Elias Métral for his strong encouragement and support to this research and for numerous suggestions and discussions on this subject. We would like to thank Gianluigi Arduini, Hannes Bartosik, Kevin Li, Giovanni Rumolo and Michael Schenk for providing valuable input and support.

\section{APPENDIX: APPLICATION TO CONVENTIONAL AND GENERALIZED WAKEFIELDS}

Although the method described in Sec. IV has been introduced to treat e-cloud effects, it can in fact be applied to different kinds of collective effects, in particular conventional wakefields, Perevedentsev's generalized wakefields, and feedback systems. For this purpose we notice that, if the dipolar forces are described by a generalized wakefield [14]:

$\Delta x^{\prime}=\frac{2 \pi R}{m_{0} \gamma v^{2}} F_{x}^{\mathrm{coh}}=\frac{e^{2}}{m_{0} \gamma v^{2}} \int d \tilde{z} \lambda_{0}(\tilde{z}) \bar{x}(\tilde{z}) W_{x}^{\mathrm{dip}}(z, \tilde{z})$,

the response functions $k_{n}(z)$ can be written as:

$$
k_{n}(z)=\frac{e^{2}}{m_{0} \gamma v^{2}} \int d \tilde{z} \lambda_{0}(\tilde{z}) h_{n}(\tilde{z}) W_{x}^{\mathrm{dip}}(z, \tilde{z}) .
$$

Conversely, if the response functions $k_{n}(z)$ are known, an equivalent generalized wakefield can be written as:

$$
W_{x}^{\operatorname{dip}}(z, \tilde{z})=\frac{m_{0} \gamma v^{2}}{e^{2} \lambda_{0}(\tilde{z})} \sum_{n=0}^{N} k_{n}(z) \frac{h_{n}(\tilde{z})}{H_{n}^{2}},
$$

as can be seen by comparing Eq. (5) against Eq. (A1). Using these expressions it is possible to verify that, for the special case in which the e-cloud quadrupolar forces are neglected, the expression of the coupling matrix in Eq. (44) coincides with the one derived in Ref. [14]. Moreover, for the special case of a conventional wakefield:

$$
W_{x}^{\mathrm{dip}}(z, \tilde{z})=W_{x}^{\mathrm{dip}}(\tilde{z}-z),
$$

in the absence of detuning effects apart from linear chromaticity, the expression of the coupling matrix in Eq. (44) coincides with the one implemented in the DELPHI code [10]. This is a consequence of the fact that in Sec. IV we have chosen the same basis functions as in Refs. [14] and in [10] to expand the perturbation on the bunch distribution.

The Vlasov method described in Sec. IV can also be used to model the effect of quadrupolar wakefields [27], in particular by computing beforehand the detuning introduced by the quadrupolar wakefield along the bunch and expressing it in the form given by Eq. (1). This feature is of particular interest since most of the available Vlasov solvers (DELPHI, MOSES [28], NHTVS [29]) do not handle this source of detuning. This possibility has been successfully tested for the case of a broad-band resonator wakefield [9] having frequency $f_{r}=2 \mathrm{GHz}$, shunt impedance $R_{s}=75 \mathrm{M} \Omega$, and quality factor $Q=1$, using the beam and machine parameters in Table I, with no e-cloud. The results obtained with the dipolar wakefield alone and with a 
(a)

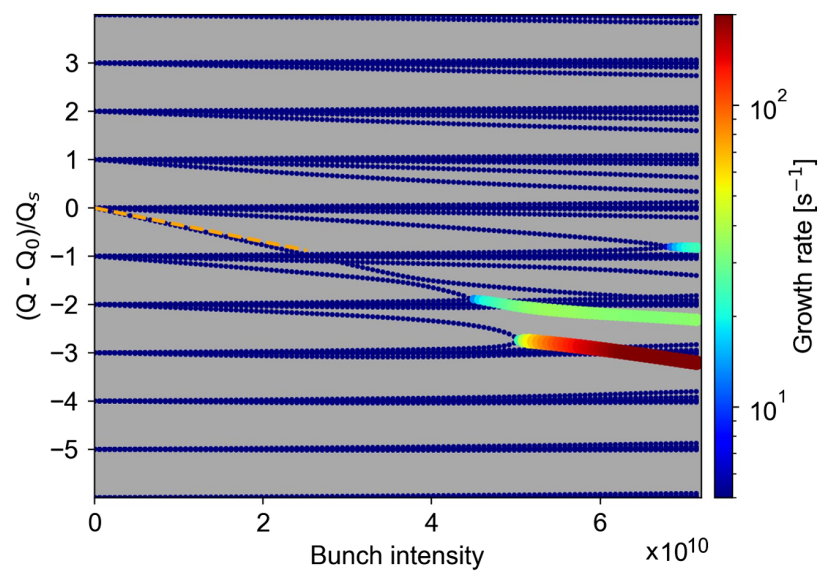

(b)

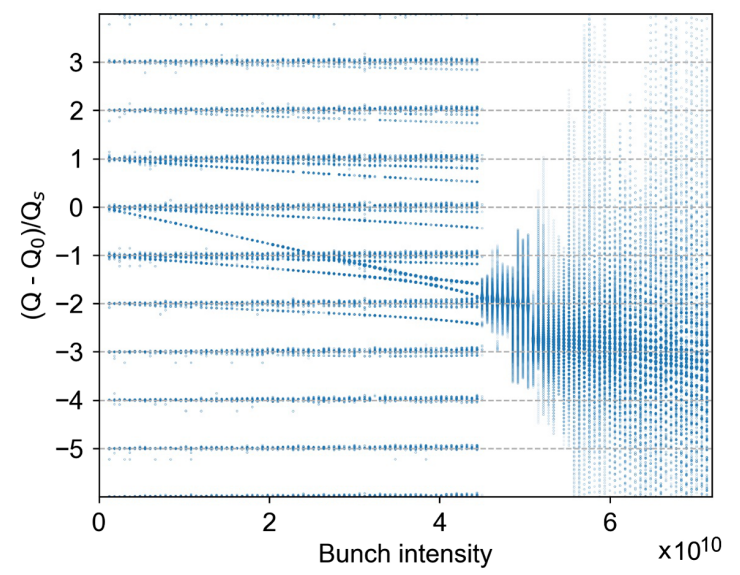

FIG. 13. (a) Frequencies of the eigenmodes computed with the Vlasov method for the case of a broad-band resonator wakefield in the absence of quadrupolar forces, $W^{\text {quad }}(z)=0$ (the color code is proportional to the corresponding growth rate). (b) Spectrum of the transverse motion for the same configuration, computed using macroparticle simulations with the PyHEADTAIL impedance module.

(a)

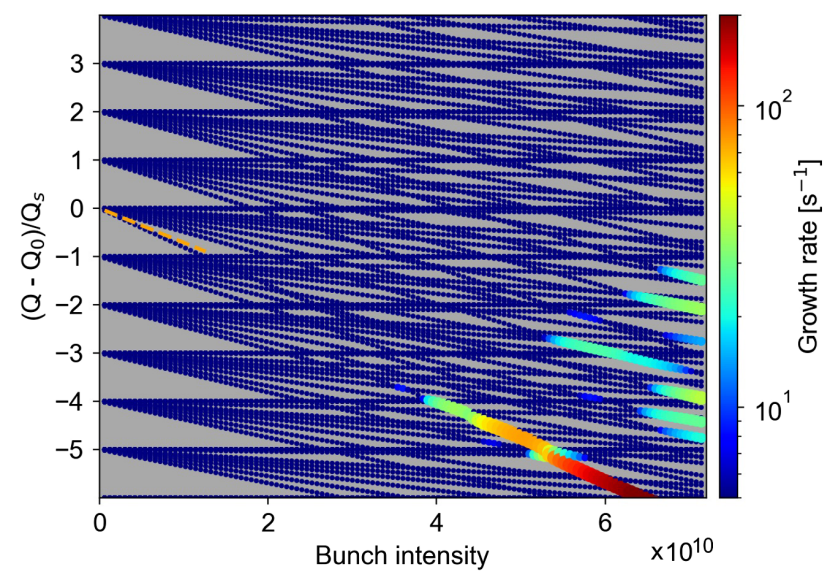

(b)

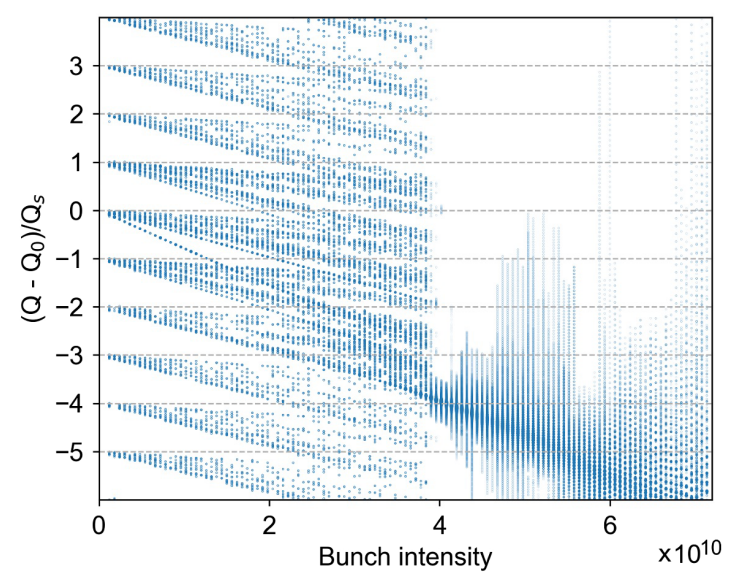

FIG. 14. Same as in Fig. 13 for the case $W^{q u a d}(z)=-W^{\operatorname{dip}}(z)$. (a) Eigenvalues from linearized Vlasov equation (b) PyHEADTAIL simulations.

(a)

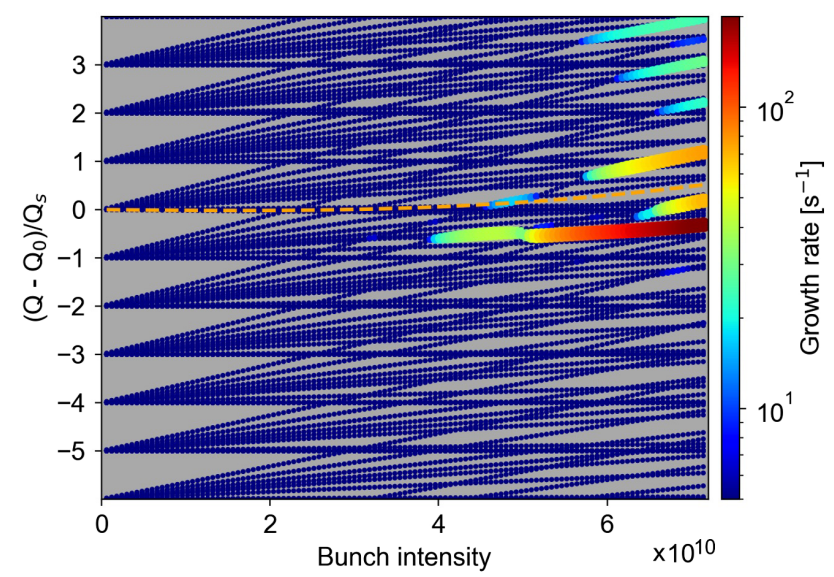

(b)

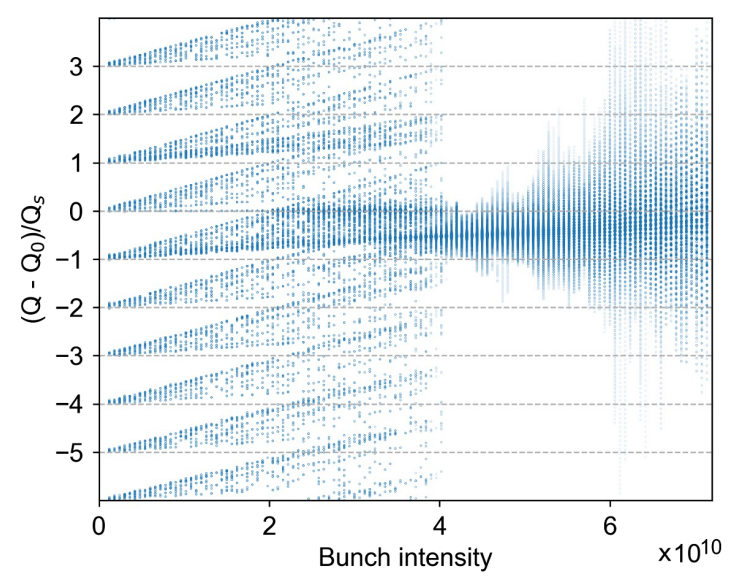

FIG. 15. Same as in Fig. 13 for the case $W^{\text {quad }}(z)=+W^{\text {dip }}(z)$. (a) Eigenvalues from linearized Vlasov equation (b) PyHEADTAIL. 
quadrupolar wakefield equal or opposite to the dipolar one are shown in Figs. 13-15. The results obtained with the Vlasov method are successfully compared against the transverse spectra from macroparticle simulations performed with PyHEADTAIL's impedance modules.

[1] Handbook of Accelerator Physics and Engineering, edited by A. W. Chao, K. H. Mess, M. Tigner, and F. Zimmermann (World Scientific, Singapore, 2013), ISBN 978-981-4415-84-2.

[2] F. Zimmermann, Electron-cloud effects in past and future machines - walk through 50 years of Electron-Cloud studies, in ECLOUD'12; 5-9 Jun 2012, La Biodola, Isola d'Elba, Italy, 15-18 Apr 2002: Proceedings (2013), pp. 917, http://cds.cern.ch/record/1567014.

[3] R. Cimino and T. Demma, Electron cloud in accelerator, Int. J. Mod. Phys. A 29, 1430023 (2014).

[4] G. Iadarola and G. Rumolo, Electron cloud effects, in ICFA Mini-Workshop on Impedances and Beam Instabilities in Particle Accelerators, 18-22 Sep 2017, Benevento, Italy: Proceedings, edited by V. Brancolini, G. Rumolo, M. Masullo, and S. Petracca (2018), pp. 49-56, https://cds .cern.ch/record/2674859.

[5] F. Zimmermann, Review of single bunch instabilities driven by an electron cloud, Phys. Rev. Accel. Beams 7, 124801 (2004).

[6] O. Domínguez, K. Li, G. Arduini, E. Métral, G. Rumolo, F. Zimmermann, and H. M. Cuna, First electron-cloud studies at the Large Hadron Collider, Phys. Rev. Accel. Beams 16, 011003 (2013).

[7] A. Romano, O. Boine-Frankenheim, X. Buffat, G. Iadarola, and G. Rumolo, Electron cloud buildup driving spontaneous vertical instabilities of stored beams in the Large Hadron Collider, Phys. Rev. Accel. Beams 21, 061002 (2018).

[8] G. Iadarola, E. Belli, K. Li, L. Mether, A. Romano, and G. Rumolo, Evolution of python tools for the simulation of electron cloud effects, in 8th International Particle Accelerator Conference (2017), p. THPAB043, https://cds.cern .ch/record/2289165.

[9] A. Chao, Physics of Collective Beam Instabilities in HighEnergy Accelerators (Wiley, New York, 1993), ISBN 9780-471-55184-3.

[10] N. Mounet, Vlasov solvers and macroparticle simulations, in ICFA Mini-Workshop on Impedances and Beam Instabilities in Particle Accelerators, 18-22 Sep 2017, Benevento, Italy: Proceedings, edited by V. Brancolini, G. Rumolo, M. Masullo, and S. Petracca (2018), pp. 77-85, https://cds.cern.ch/record/2674107.

[11] G. Rumolo and F. Zimmermann, Electron cloud simulations: beam instabilities and wakefields, Phys. Rev. Accel. Beams 5, 121002 (2002).

[12] M. Schenk, A. Grudiev, K. Li, and K. Papke, Analysis of transverse beam stabilization with radio frequency quadrupoles, Phys. Rev. Accel. Beams 20, 104402 (2017).

[13] K. Ohmi, F. Zimmermann, and E. Perevedentsev, Wakefield and fast head-tail instability caused by an electron cloud, Phys. Rev. E 65, 016502 (2001).
[14] E. Perevedentsev, Head-tail instability caused by electron cloud, in ECLOUD'02: Mini-workshop on electron-cloud simulations for proton and positron beams, CERN, Geneva, Switzerland, 15-18 Apr 2002: Proceedings (2002), pp. 171194, https://cds.cern.ch/record/585578.

[15] R. J. Macek, A. A. Browman, J. E. Ledford, M. J. Borden, J. F. OHara, R. C. McCrady, L. J. Rybarcyk, T. Spickermann, T. J. Zaugg, and M. T. Pivi, Electron cloud generation and trapping in a quadrupole magnet at the Los Alamos Proton Storage Ring, Phys. Rev. Accel. Beams 11, 010101 (2008).

[16] A. A. Vlasov, On the kinetic theory of an assembly of particles with collective interaction, Russ. Phys. J. 9, 25 (1945).

[17] N. Mounet, Direct Vlasov solvers, in Proc. of the CASCERN Accelerator School on Numerical Methods for Analysis, Design and Modelling of Particle Accelerators, Thessaloniki, Greece, 11-23 November 2018, edited by H. Schmickler (CERN, Geneva, 2020), CERN Accelerator School Proceedings, https://arxiv.org/abs/2006.09080.

[18] M. Schenk, X. Buffat, K. Li, and A. Maillard, Vlasov description of the effects of nonlinear chromaticity on transverse coherent beam instabilities, Phys. Rev. Accel. Beams 21, 084402 (2018).

[19] Y.-H. Chin, Hamiltonian formulation for transverse bunched beam instabilities in the presence of betatron tunespread (1985), https://cds.cern.ch/record/160217/files/ 198507010.pdf.

[20] G. Besnier, D. Brandt, and B. Zotter, The transverse mode coupling instability in large storage rings, Particle Accelerator, 17, 51 (1985), https://cds.cern.ch/record/154782/ files/p51.pdf.

[21] M. Abramowitz, I. A. Stegun, and R. H. Romer, Handbook of mathematical functions with formulas, graphs, and mathematical tables, Am. J. Phys. 56, 958 (1988).

[22] Y.-H. Chin, Transverse mode coupling instabilities in the SPS (1985), https://cds.cern.ch/record/157995/files/ 198503163.pdf.

[23] R. Bartolini and F. Schmidt, A computer code for frequency analysis of non-linear betatron motion, CERN Technical Report No. SL-Note-98-017-AP, 1998, https:// cds.cern.ch/record/702438.

[24] E. Metral and M. Migliorati, Vlasov solvers and simulation code analysis for mode-coupling instabilities in both longitudinal and transverse planes, in ICFA mini-Workshop on "Mitigation of Coherent Beam Instabilities in particle accelerators" MCBI 2019, proceedings (to be published).

[25] A. Savitzky and M. J. Golay, Smoothing and differentiation of data by simplified least squares procedures, Anal. Chem. 36, 1627 (1964).

[26] A. Burov, Three-beam instabilily in the LHC, ICFA Beam Dyn. Newslett. 69, 264 (2016), https://cds.cern.ch/record/ 1522567.

[27] E. Metral, X. Buffat, and G. Rumolo, Transverse modecoupling instability in the presence of detuning impedance, https://cds.cern.ch/record/2714848.

[28] Y. H. Chin, MOde-coupling Single bunch instability in an Electron Storage ring, CERN Technical Report No. CERNLEP-TH-88-05, 1988, https://cds.cern.ch/record/187253.

[29] A. Burov, Nested head-tail Vlasov solver, Phys. Rev. Accel. Beams 17, 021007 (2014). 